\title{
SIMULATION MODELLING OF NANOPARTICLE ASSEMBLIES FORMED BY SPRAY DEPOSITION
}

\author{
Tomás H. Maul \\ Computer Science \\ The University of Nottingham Malaysia Campus \\ Jalan Broga, 43500 Semenyih, \\ Malaysia \\ E-mail: Tomas.Maul@nottingham.edu.my
}

Yuying Yan

Faculty of Engineering

The University of Nottingham

University Park, Nottingham

UK

E-mail: Yuying.yan@nottingham.ac.uk

\section{KEYWORDS}

Nanoparticle Assemblies, Roughness, Contact Angle, Superhydrophobicity, Retinal Prosthesis.

\begin{abstract}
This paper describes a simulation model of nanoparticle assemblies formed by spray deposition. The simulation deposits nanoparticles via a semi-mechanistic process, which in spite of its simplicity generates morphologies of considerable complexity. The experiments reveal several relationships between nanoparticle parameters such as their relative proportions in mixtures, and resulting surface properties, such as roughness and superhydrophobicity. According to the simulation results, for mixtures involving nanoparticles with diameters $7 \mathrm{~nm}$ and $14 \mathrm{~nm}$, a relative proportion of $40 \%$ vs. $60 \%$ respectively, produces the surfaces with largest contact angle.
\end{abstract}

\section{INTRODUCTION}

The current paper is concerned with the simulation modelling of nanoparticle assemblies formed by spray deposition. The study was initially motivated by the need to develop new retinal prostheses with increased resolution and improved information processing. In the context of information processing we have been developing retinal models in order to better understand the structure and function of the human retina (Maul et al. 2010). In the context of increasing prosthesis resolution, we are currently looking at nanotechnological solutions. The general idea is that by increasing the area of electrodes via complex surface structures we can decrease impedance and therefore decrease the diameter of electrodes (Kim et al. 2010). Currently we are investigating complex hierarchical structures that are formed by spraying mixtures of nanoparticles of different sizes onto glass substrates (Gao et al. 2010). Although our underlying motivation resides in the design of retinal prosthesis, our simulation

\author{
Andrzej Bargiela \\ Computer Science \\ The University of Nottingham \\ Jubilee Campus, Wollaton Road, Nottingham \\ UK \\ E-mail: Andrzej.Bargiela@nottingham.ac.uk \\ Nan Gao \\ Faculty of Engineering \\ The University of Nottingham \\ University Park, Nottingham \\ UK \\ E-mail: laxng2@nottingham.ac.uk
}

model can be used more generally, and in fact, in this paper, we will focus on investigating its surface and hydrophobic properties.

Nanoparticles consist of particles whose diameters lie in the nano-scale $\left(10^{-7}-10^{-9} \mathrm{~m}\right)$. They can be composed of different elements such as gold, silver, silicon dioxide, titanium and many others. Because of the unique electrical (and other) phenomena that occur at this level (which can differ significantly from the atomic, micro and macro scales), nanoparticles hold great promise for several different applications, e.g.: electronics ( $\mathrm{Fu}$ et al. 2005), energy (Bisquert 2008), health (Hawkins et al. 2008), and so on. Nanoparticle assemblies can be formed via different methods, which result in varied structural and functional forms. As already mentioned we focus on multilayered nanoparticle assemblies on glass substrates formed via spray deposition.

Nanoparticle assemblies formed in such a manner can exhibit a host of different properties. In this paper we will concentrate on their tendency to form complex hierarchical structures and how this affects wettability properties. Figure 1 depicts an example of such a surface as measured by Atomic Force Microscopy (AFM). The wettability of a surface can be seen as the inverse of the contact angle (CA) (Zisman 1964), where the latter is defined as the angle between the liquid/vapour interface and the contacted solid surface. By definition, when the CA is less than or equal to $90^{\circ}$ the surface is hydrophilic, when it is larger than $90^{\circ}$ but less than or equal to $150^{\circ}$ it is hydrophobic, and when it is larger than $150^{\circ}$, it is super-hydrophobic. 


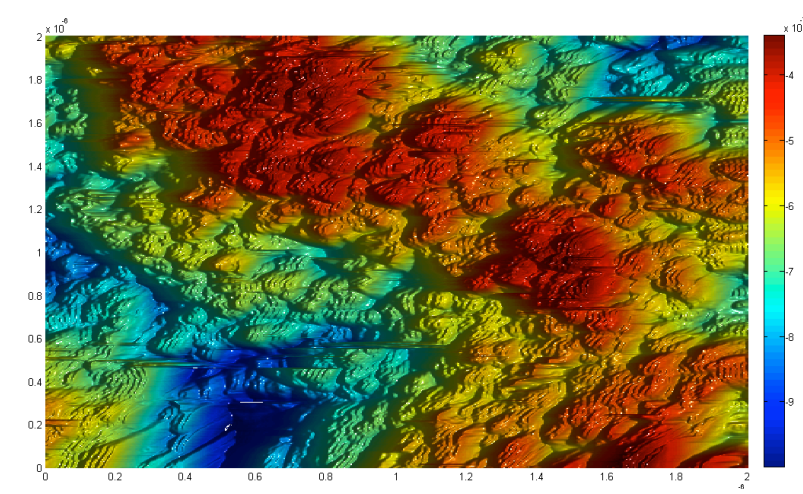

Figure 1. AFM image: nanoparticle assembly surface.

As much as there are many interesting discoveries and applications arising from research into novel nanomaterials everyday, many questions regarding their physical, chemical, electric, magnetic and optical properties remain unanswered. For example, with regards to the spray deposition method, it is not fully known to what extent and in what ways, nanoparticles, interact with each other after making contact with the substrate. This is one of the reasons why simulation studies can be very helpful (Barnard 2010) (Suvakov and Tadic 2010). Through simulation we can experiment with different theories regarding the deposition process and test them with respect to the available empirical data.

So far, to the best of our knowledge no semimechanistic simulation of spray deposition has been developed that allows users to manipulate multiple nanoparticles types, in different proportions, and deposited in one or more stages. This type of simulation model can save a considerable amount of time/money by predicting the most effective mixtures and spray procedures. We have also implemented functions that extract and analyze the resulting surfaces in order to generate estimates of their hydrophobic and electrical (not reported) properties.

The main objectives of this paper are to introduce a new simulation model of a nanoparticle assembly formation process, to demonstrate how the model can be used by nanoengineers in the experimental process, and to discuss several early results, which at this stage, although they generate more questions than answers, confirm the suspicion that even simple models of nano assembly processes can generate complex and unexpected patterns.

The next three sections will provide details of the simulation model, and will present several sets of results which will be briefly discussed followed by an outline of future work.

\section{METHODS}

The model allows the user to define different nanoparticle assemblies by controlling several parameters, the most important of which are the number of nanoparticle types in the mixture and the sizes and relative quantities of each nanoparticle type. The simulated deposition process is summarized in Table 1.

The simulated deposition process starts by generating a cube of the right dimensions, which will contain the deposited nanoparticles. At each iteration, a particle is chosen probabilistically. The probability of a particle being chosen corresponds to its relative proportion in the mixture. The $x y$ coordinate at which the particle enters the cube is also chosen randomly (uniform distribution). After a particle and a coordinate have been chosen, the particle is projected perpendicularly to the surface of the substrate until it either collides with the substrate or another particle. If it collides with the substrate then the simulation proceeds to the next particle. If it collides with another particle, the angle of the line between the centres of the colliding particles relative to the $x y$ plane is compared to a user-specified threshold (hereafter referred to as bounce angle). Refer to Figure 2 for a diagram of particle and threshold angles. If the particle angle is larger than the threshold (case A in Figure 2) then the particle remains where it is and the simulation proceeds to the next particle. If the angle is smaller or equal to the threshold (case B in Figure 2) then the particle bounces off and continues to fall until it collides again. It will continue in this bounce/fall manner until it gets stuck, hits the substrate or collides with a particle at an angle larger than the threshold. Refer to Figure 3 for a diagram of this bounce/fall behaviour: in case $\mathrm{A}$ the particle remains at the point of collision; in case B the particle keeps bouncing and falling until it hits the substrate; in case $\mathrm{C}$ the particle bounces off the first particle and then bounces back and so is considered to be stuck.

Table 1. Summarized deposition process.

\begin{tabular}{|c|c|}
\hline $\mathbf{1}$ & Initialize spraying space \\
\cline { 1 - 1 } $\mathbf{2}$ & Initialize particles \\
\cline { 1 - 1 } $\mathbf{3}$ & For i=1 to number of particles \\
Select a particle probabilistically \\
$\mathbf{4}$ & Sele \\
\cline { 1 - 1 } $\mathbf{5}$ & Select an $x y$ coordinate randomly \\
\cline { 1 - 1 } $\mathbf{6}$ & Let the particle fall until it collides \\
\cline { 1 - 1 } $\mathbf{7}$ & If the collision is with the substrate then \\
\cline { 1 - 1 } $\mathbf{8}$ & continue with the next particle (4) \\
\cline { 1 - 1 } $\mathbf{9}$ & If the collision angle is larger than the threshold \\
\cline { 1 - 1 } $\mathbf{1 0}$ & continue with the next particle (4) \\
\cline { 1 - 1 } $\mathbf{1 1}$ & Otherwise \\
\cline { 1 - 1 } $\mathbf{1 2}$ & Bounce and continue down until trapped \\
\cline { 1 - 1 } $\mathbf{1 3}$ & End For \\
\hline
\end{tabular}




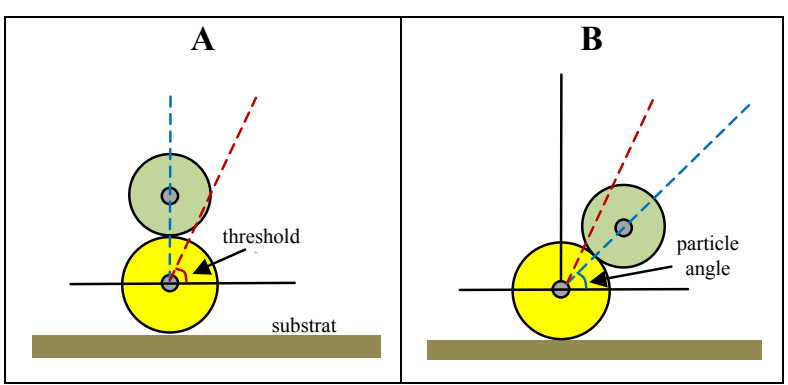

Figure 2. Particle and threshold angles.

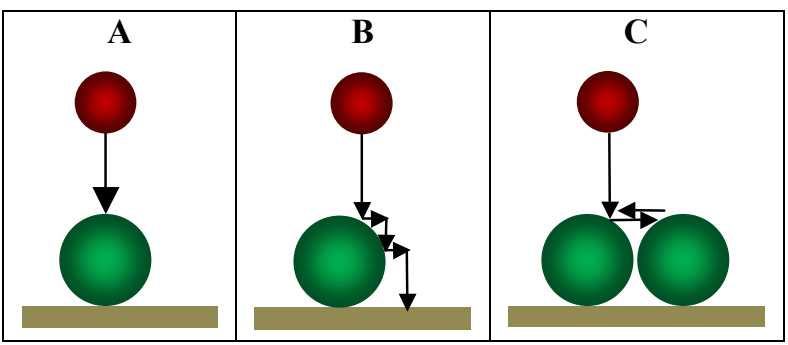

Figure 3. Collision behaviours.

Once all the particles have been deposited the simulation proceeds to extract the top surface of the nanoparticle assembly. This is done by sampling multiple $x y$ coordinates and determining the topmost coordinates of the nanoparticle assembly at those points. After collecting these samples the rest of the surface is constructed using cubic interpolation. Figure 4 depicts an example of a surface estimated in this manner.

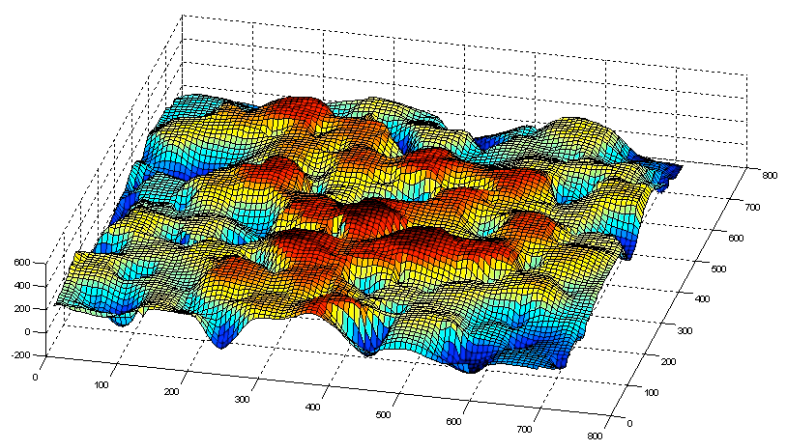

Figure 4. A nanoparticle assembly surface.

Following this, the extracted surface is analyzed in order to estimate several properties, the most important of which are roughness, and wet-dry and rough-wet ratios. The last two properties are relevant in the context of hydrophobicity studies. Roughness $r$ is defined as the ratio of the external surface area of the nano-particle assembly to the projected area on the substrate. Since not all surfaces may be wetted for different topologies of nanoparticle assemblies we define also the wet-dry ratio $w d$ as a ratio of the external surface of the nanoparticle assembly that is wetted to the total surface of the assembly. Similarly we define the rough-wet ratio $r w$ as a ratio of the wetted surface to the orthogonal projection of this surface as depicted in Figure 5. In order to calculate the wet-dry and rough-wet ratios we first need to estimate the contact area between a hypothetical droplet and the nanoparticle assembly surface. In Figure 5 (top) the estimated contact regions between a droplet and the surface are depicted by darker grayscale intensities. In our current implementation slicing of the surface peaks is performed at height $b$ calculated as $b=v+l(p-v)$ where $p$ is the highest peak, $v$ is the lowest valley and $l$ is a (physically informed) parameter in the interval $[0,1]$. Note that because the above three ratios divide area by area, they are all unitless.
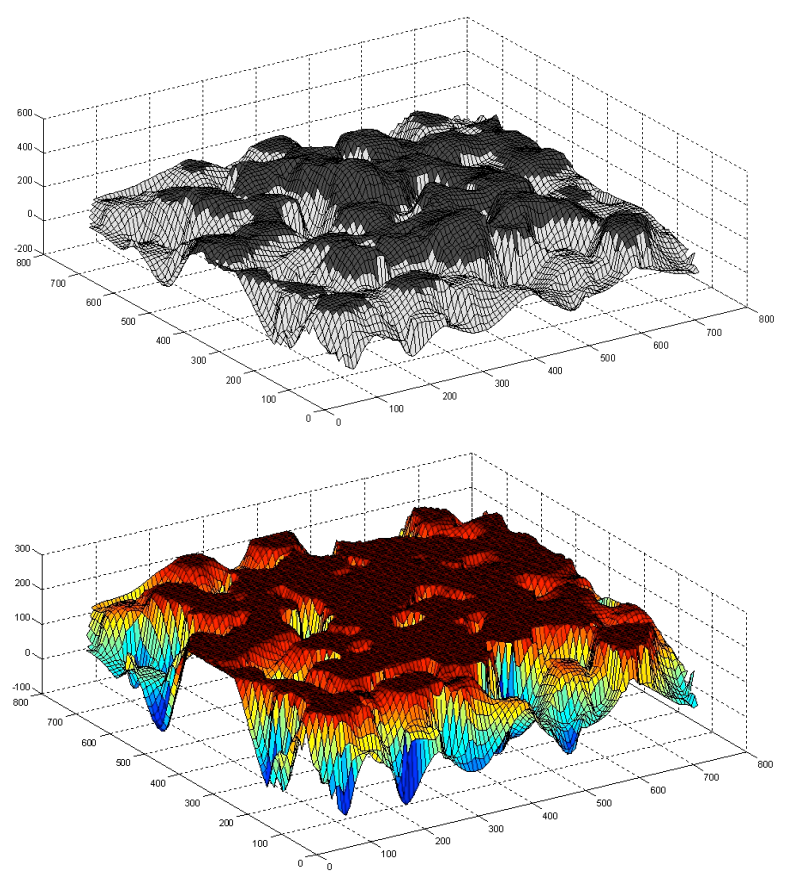

Figure 5. Droplet and surface contact area.

The rough surfaces that are fully wetted, i.e. those that have $w d=1$ and $r>1$, increase their hydrophobicity in proportion to their roughness according to the formula proposed by Wenzel (Marmur, 2003),

$$
\cos \theta^{*}=r \cos \theta
$$

where $\theta^{*}$ is the apparent contact angle which corresponds to the stable (minimum free energy for the system) and $\theta$ is the Young's contact angle derived for an ideal surface from the balance of surface tension forces between the solid-liquid, liquid-vapour and solidvapour.

For the rough surfaces that are not fully wetted, i.e. those that have $0<w d<1$ and $r>1$, the Wenzel equation does not describe correctly the increase of the hydrophobicity because of its omission of the effect of the liquid-vapour surface tension in the areas that that trap the vapour phase. The spectrum of cases defined by different wet-dry ratio, $w d$, are described by the CassieBaxter equation (Marmur, 2003), 


$$
\cos \theta^{*}=r w w d \cos \theta+w d-1
$$

It is clear that for full wetting, i.e. when $w d=1$, the $r w=r$ and the equation (2) becomes equivalent to (1). The transition from the Cassie state to the Wenzel state occurs at the critical contact angle, $\theta_{C}$, evaluated as follows

$$
\cos \theta_{C}=(w d-1) /(r w-1)
$$

under the assumption that $\mathrm{rw}>1$. If the contact angle is from $\pi / 2$ to $\theta_{C}$ the wetting is described by Wenzel model (1) and if the contact angle is greater than $\theta_{C}$ the wetting is described by the Cassie-Baxter model (2).

Due to the stochastic nature of both particle and entrycoordinate selection, most of our experiments involved running 50 tests for each parameter configuration, unless otherwise specified.

\section{RESULTS}

The first set of experiments involved creating assemblies of 300 nanoparticles of a single type (i.e. size). Figure 6 depicts experiments where we systematically varied the bounce angle (i.e. $70^{\circ}, 80^{\circ}$ or $90^{\circ}$ ) and the particles involved (i.e. particles with diameters of $7 \mathrm{~nm}, 14 \mathrm{~nm}$ or $50 \mathrm{~nm}$ ). Particle sizes were chosen in order to conform to the physical experiments conducted in (Gao et al. 2010). Each experimental condition was run 50 times in order to average out the effects of stochastic particle placement. The figure depicts mean roughness values for each condition. Notice how roughness is directly proportional to bounce angle and inversely proportional particle size. Refer to Figure 7 to get an intuitive notion of the effect of bounce angle on the morphology of assembly structures.

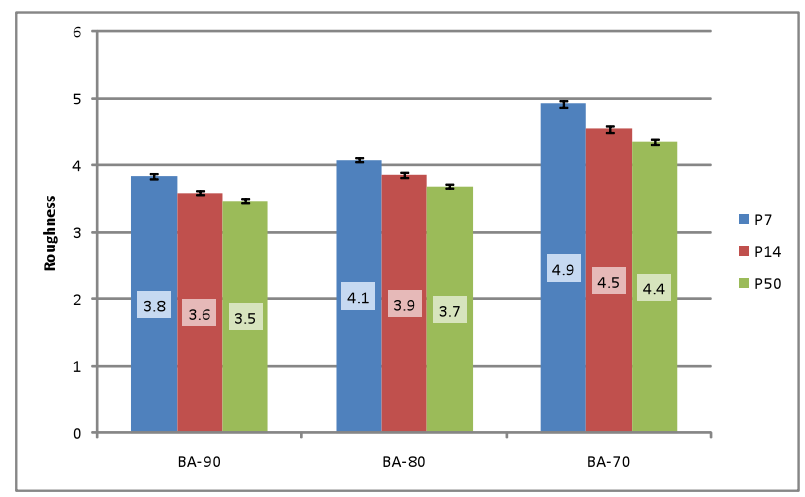

Figure 6. Particle type, bounce angle and roughness.
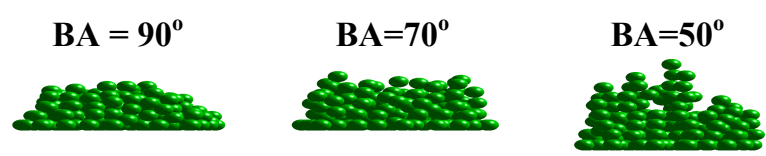

Figure 7. Bounce angles and particle configurations.
Still for the same set of experiments we also estimated water droplet contact angles (based on wet-dry and rough-wet ratios). According to

Figure 8, as expected contact angle is directly proportional to bounce angle. Within each bounce angle, the relationship between particle type and contact angle is not monotonic. This can be partially explained by looking at Figure 9 and Figure 10, which represent the relationships between bounce angle and particle type on one hand, and wet-dry and rough-wet ratios respectively, on the other.

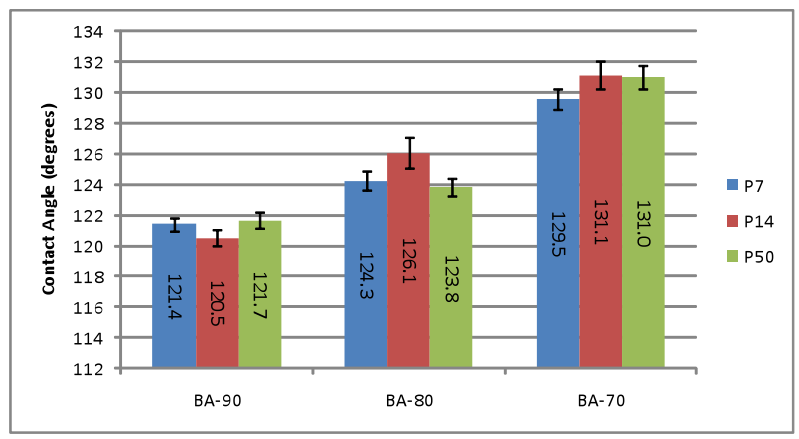

Figure 8. Particle type, bounce angle and contact angle.

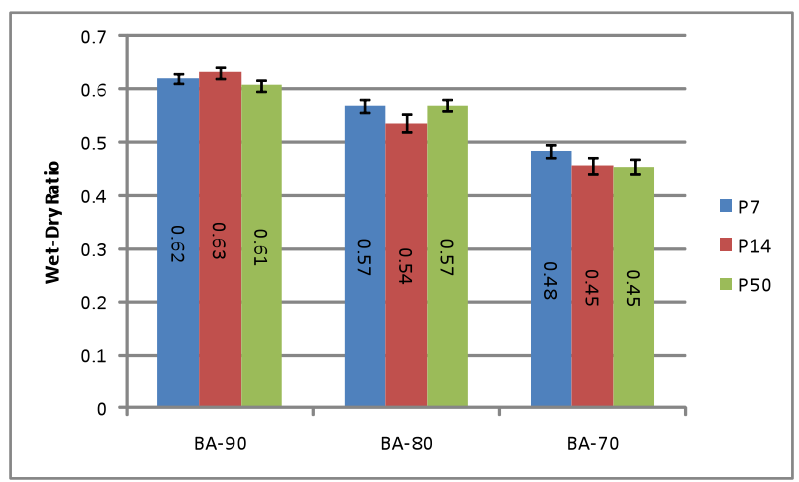

Figure 9. Particle type, bounce angle and wet-dry ratio.

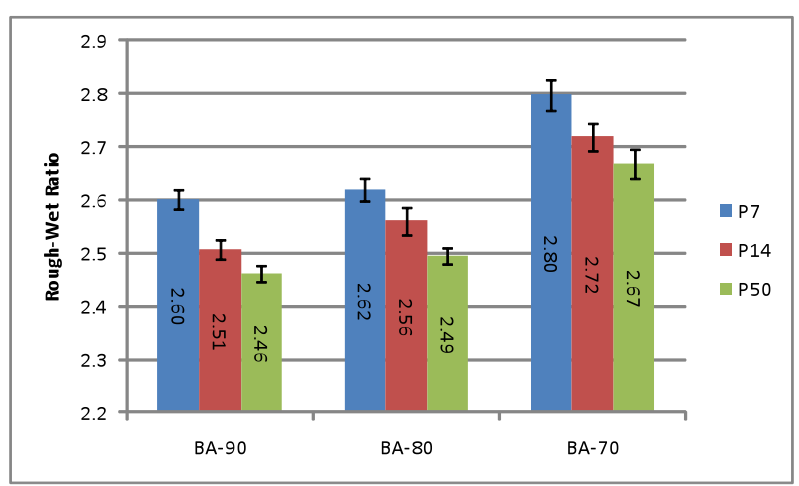

Figure 10. Particles, bounce angle and rough-wet ratio.

Another set of experiments (from Figure 11 to Figure 14) was conducted in order to study the properties of nanoparticle assemblies consisting of mixtures of two different particle types (i.e. $7 \mathrm{~nm}$ and $14 \mathrm{~nm}$ diameter particles). Nanoparticle proportions in the mixtures 
were varied from $0 \%$ to $100 \%$ in $10 \%$ increments, where for example, a P7 proportion of $x \%$ signifies that $x \%$ of the total assembly volume (in our experiments $5 d^{2}$, where $d$ represents the length of the substrate size) is occupied by $\mathrm{P} 7$ particles and $(100-x) \%$ is occupied by P14 particles. All cases correspond to bounce angles of $90^{\circ}$. Because of the larger substrates used in these experiments (i.e. side $=1,000 \mathrm{~nm}$ ), we reduced the number of tests per configuration down to 5 .

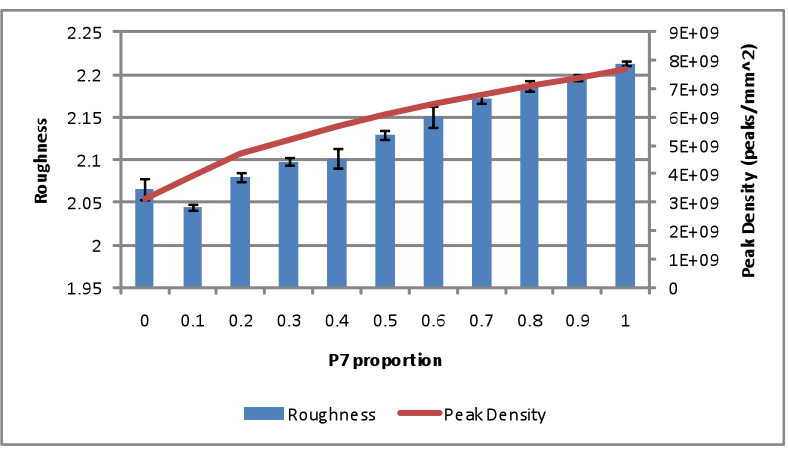

Figure 11. The effect of particle proportion on surface roughness and peak density.

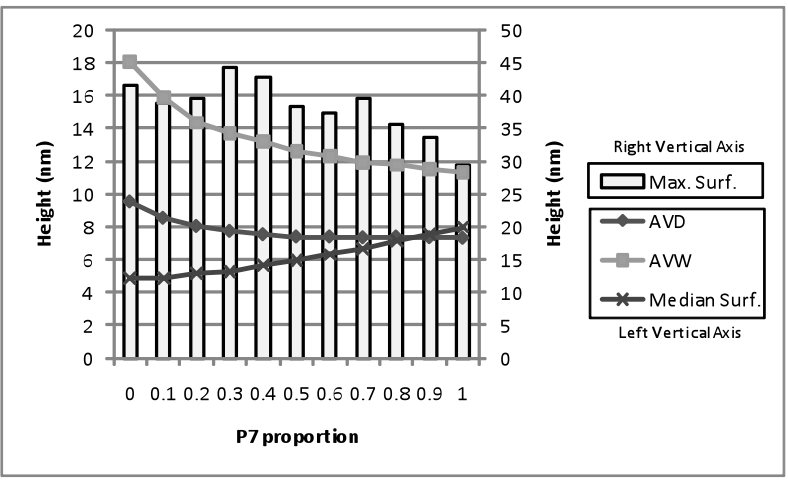

Figure 12. Particle proportion and surface properties.

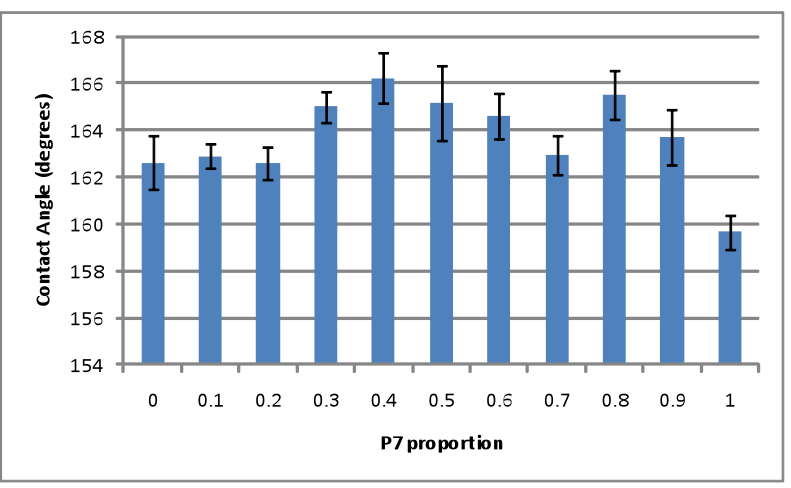

Figure 13. Effect of particle proportion on contact angle.

$P 14 / P 50=20 / 80$

$\mathbf{P} 14 / \mathbf{P 5 0}=\mathbf{8 0} / 20$
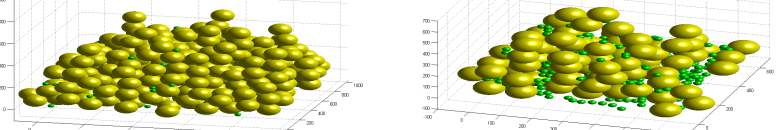

Figure 14. Two different particle proportions.

Figure 11 depicts mean roughness and peak densities for different proportions of P7 particles relative to P14 particles. Figure 12 depicts the effect of particle proportion on several other surface properties, namely: average valley depth (AVD) (average height difference between valleys and surrounding peaks), average valley width (AVW) and median and maximum surface height. Figure 13 depicts the effect of particle proportion on contact angle (Cassie-Baxter). In these experiments, Young's intrinsic contact angle was set to $95^{\circ}$. Figure 14 depicts examples of nanoparticle assemblies for two different particle proportions.

\section{DISCUSSION}

One of the more obvious and expected results includes the direct proportionality between bounce angle and surface roughness. As the bounce angle decreases the probability of a particle sticking to another (i.e. not bouncing) increases. This increased stickiness leads to the formation of vertically elongated structures (as we can see in Figure 7), which in turn contributes to increased surface roughness. Consequently, this relationship can be seen in all of the graphs in the results section.

Another unsurprising result is concerned with the roughness of assemblies consisting of single nanoparticle types. As can be seen in Figure 6, roughness is inversely proportional to particle size. One way of understanding this is to consider two sets of nanoparticles of different sizes, where each set makes up the same total volume. The smaller nanoparticles can be configured into more complex shapes than the larger nanoparticles, and therefore can lead to larger surface areas. The same surface complexity argument applies to our results.

The relative advantage of smaller particles (in terms of roughness) is also observed in binary mixtures (i.e. assemblies consisting of two particle types). Figure 11 shows how roughness increases in a manner that is directly proportional to the percentage of volume that is comprised by $\mathrm{P} 7$ particles. As the same graph illustrates, this can be explained by a parallel increase in peak density. It is interesting to see in Figure 12 that this increase in peak density is sufficient to compensate for a corresponding decrease in valley depth. Figure 12 also illustrates other unsurprising principles such as the fact that both valley width and maximum surface height are inversely proportional to the proportion of $\mathrm{P} 7$ and the fact that the median surface height is directly 
proportional to the $\mathrm{P} 7$ proportion. These results all seem to point to the fact that there is no "mixture advantage", i.e.: there is no combination of two particle types (of different sizes) that confers a roughness advantage relative to the assemblies formed by each particle type individually. According to the results, in order to maximize roughness all we need to do is maximize the proportion of smaller particles. In other words we can do away with the larger particles.

But does this simple finding translate directly to hydrophobicity properties? According to Figure 13 it does not. According to this figure mixtures of particles (e.g. $\mathrm{P} 7=0.3$ and $\mathrm{P} 7=0.4$ ) have larger contact angles than assemblies consisting of single particles (i.e. $\mathrm{P} 7=0$ and $\mathrm{P} 7=1)$. This is partially explained by Figure 15 : notice how the left-hand side graph (wet-dry ratio) is approximately a mirror image of Figure 13. In conclusion, at least in terms of contact angle (CassieBaxter), there does seem to be a "mixture advantage" which correlates well with some of the findings reported in Gao et al. 2010.
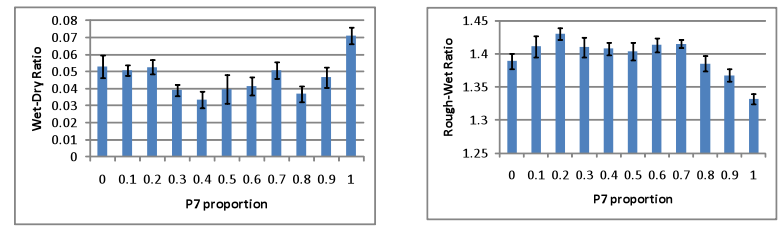

Figure 15 . Wetting ratios for $90^{\circ}$ bouncing angle.

From the results we can see that even with a simple nanoparticle deposition model, it is not easy to know a priori what particle combinations produce the largest contact angles. If nothing else, the results show us that parameters such as particle proportions and bounce angles interact in interesting and non-obvious ways. Once sufficient empirical data is available, we will be able to fine-tune and validate the deposition model, and we expect that simulation-based experiments such as these will help experimenters save time and money by recommending an optimal set of experiments to conduct.

In the near-future we expect to: 1) fine tune and validate the model with new empirical data, 2) extend the model so that it can estimate the electrical properties of nanoparticle assemblies and 3) run extensive optimization experiments with the separate goals of maximizing both hydrophobicity and conductivity. In the relatively distant future we hope that the nanoparticle assemblies found with the help of these simulation studies will be incorporated into novel microelectrode arrays to be applied in neural prostheses. By creating surfaces with increased roughness, the impedance of electrodes can be decreased, allowing the electrodes to be miniaturized further, finally contributing to a much needed increase in prosthesis resolution.

\section{REFERENCES}

Maul, T.H., Bargiela, A. And Ren, L.J. 2010 "Cybernetics of Vision Systems: Toward an Understanding of Putative Functions of the Outer Retina." IEEE Transactions on Systems, Man and Cybernetics, Part A: Systems and Humans, No. 99.

Kim, J.H., Kang, G., Nam, Y. and Choi, Y.K. 2010 "Surfacemodified microelectrode array with flake nanostructure for neural recording and stimulation." Nanotechnology, Vol. 21.

Gao, N., Yan, Y.Y., Chen, X.Y. and Zheng, X.F. 2010. "Superhydrophobic Composite Films Based on THS and Nanoparticles." Journal of Bionic Engineering, Vol. 7, 5966.

Fu, L., Liu, Z., Liu, Y., Han, B., Hu, P., Cao, L. and Zhu, D. 2005 "Beaded cobalt oxide nanoparticles along carbon nanotubes: towards more highly integrated electronic devices." Advanced Materials, 17:2, 217-221.

Bisquert, J. 2008 "Physical electrochemistry of nanostructured devices" PCCP: Physical Chemistry Chemical Physics, 10:1, 49-72.

Hawkins, M.J., Soon-Shiong, P. and Desai, N. 2008 "Protein nanoparticles as drug carriers in clinical medicine" Advanced drug delivery reviews, 60:8, 876-885.

Zisman, W.A. (1964) "Relation of the equilibrium contact angle to liquid and solid constitution" Advances in Chemistry Series, Vol. 43.

Barnard, A.S. 2010. "Modelling of nanoparticles: approaches to morphology and evolution" Reports on Progress in Physics, 73:8.

Suvakov, M. and Tadic, B. 2010. "Modeling collective charge transport in nanoparticle assemblies" Journal of Physics: Condensed Matter, 22:16.

Marmur, A, 2003. "Wetting of hydrophobic rough surfaces: To be heterogeneous or not to be", Langmuir 19, 83438348. 


\section{AUTHOR BIOGRAPHIES}

TOMAS H. MAUL was born in Madeira, Portugal and did a BSc. in Biological Psychology at the University of St. Andrews, an MSc. in Computer Science at Imperial College and a PhD. in Computational Neuroscience at the University of Malaya. He worked for two years at MIMOS Bhd. as a Senior Researcher in the fields of Pattern Recognition and Computer Vision. $\mathrm{He}$ is currently an Assistant Professor at the University of Nottingham Malaysia Campus, where he conducts research in the areas of Neural Computation, Optimization and Computer Vision. His e-mail address is Tomas.Maul@nottingham.edu.my and his Web-page is http: //baggins.nottingham.edu.my/ kcztm/

ANDRZEJ BARGIELA is Professor of Computer Science at the University of Nottingham. Until recently he was Director of Computer Science at the University of Nottingham, Malaysia Campus. He is a member of the Automated Scheduling and Planning research group in the School of Computer Science at the University of Nottingham. Since 1978 he has pursued research focused on processing of uncertainty in the context of modelling and simulation of various physical and engineering systems.

YUYING YAN is a Reader in Thermofluids Engineering at the University of Nottingham. He is a member of Energy \& Sustainability Research Division in Faculty of Engineering. His research covers wideranging aspects of thermofluids flow and heat transfer encountered in engineering thermal systems and the building services. He is also doing multi-disciplinary research on biomimetics of natural functional surfaces with fluids interactions.

NAN GAO BSc, MSc, is currently a third year $\mathrm{PhD}$ researcher at Faculty of Engineering, University of Nottingham. Nan Gao obtained his first degree in Mechanical \& Agricultural Engineering and MSc in Mechanical \& Bionic Engineering at Jilin University, China. His $\mathrm{PhD}$ research under Prof. Yan's supervison has been concentrating on the fabrication and phenomena related to superhydrophobic surfaces. He is interested in the application of nanoparticles to achieving superhydrophobicity by controlling the micro $\&$ nano structure. 